\title{
RECENTES MUDANÇAS PROPOSTAS NA AVALIAÇÃO ANTROPOMÉTRICA DO ESTADO NUTRICIONAL INFANTIL: UMA AVALIAÇÃO CRÍTICA
}

Carlos Augusto Morteiro *

\begin{abstract}
MONTEIRO, C. A. Recentes mudanças propostas na avaliação antropométrica do estado nutricional infantil: uma avalią̧ão crítica. Rev. Saúde públ., S. Paulo, 18:56-63, 1984.
\end{abstract}

RESUMO: Modificações na avaliação do estado nutricional infantil, pur intermédio da antropometria, vem sendo recentemence propostas em puolicações nacionais e internacionais de grande prestígio e velculação. Tais modificações envolvem a substituição do padrão antropométrico "Harvard" e a substituição dos critérios da "Classificação de Goinez" baseados no peso e idade da criança. A luz da teoria que ampara o diagnóstico antropométrico dc estado nutricional, são criticamente analisadas tanto as objeções levantadas contra o método diagnóstico tradicional quanto às novas proposições formuladas. Da análise, depreende-se que: a) a adoção de novos pađrões ("Santo André Classe IV" e "NCHS") se justifica plenamente, ainda que pouca repercussão prática possa esperar-se da modificaçâo; b) a adoção de percentis do padrão para expressar niveis críticos é aperfeiçoamento importante quando um amplo espectro de idades é examinado ou quando mais de um indicador antropométrico é cogitado; c) o rebaixamento extremo de níveis antropométricos críticos e a implícita preocupação unilateral com a especificidade do diagnóstico não encontram justificativa em nosso meio onde a desnutrição não é evento raro; d) em determinadas idades, a observação continua do indicador peso/idade pode resultar mais vantajosa do que a introdução de novos indicadores como altura/idade e peso/altura. Da análise realizada, depreende-se também que a propriedade de critérios antroponétricos na avaliação nutricional não pode ser devidamente apreciada sem que sejam explicitados o propósito da avaliação e o possível nivel de endemicidade da desnutrição na população a ser examinada.

UNITERMOS: Antropometria. Crianças. Estado nutricional. Desnutrição'

\section{INTRODUÇĀO}

O controle do crescimento é consensualmente aceito como instrumento de utilidade singular na avaliação do estado de saúde e nutrição da criança, sendo decisivo no diagnóstico da desnutrição protéico-calórica (DPC) e fornecendo importantes subsidios no diagnóstico de outras deficiências nutri. cionais e mesmo do próprio estado geral cit saúde.
O exame antroponétrico como método de escolha para o diagnóstico da DPC justifica-se não só em função de aspectos ligados a praticabilidade e custo, mas também porque na DPC o retardo do crescimento é sistemático e freqüentemente precede as demais manifestações da deficienticia. Exame de sinais clinicos e exames bioquimicos, procedimentos de escolha para 0

\footnotetext{
* Do Departamento de Nutrição da Faculdade de Saúde Pública da Universidade de São Paulo - Av. Dr. Arnaldo, 715 - 01255 - São Paulo, SP - Brasil .
} 
MONTEIRO, C.A. Recentes mudanças propostas na avaliação antropométrica do estado nutricional infantil: uma avaliação crítica. Rev. Saúde públ., S. Paulo, 18:56-63, 1984.

diagnóstico das deficiências de micro-nutrientes como Ferro, Iodo, Vitamina A entre outros, não apresentam a mesma utilidade em relação à deficiência de proteínas e calorias, uma vez que, regra geral, sinais clínicos e alterações bioquimicas - como edema e hipoalbuminemia por exemplo não são sistemátıcos na $\mathrm{DPC}$, além de, com certa frequiência, ocorrerem tardiamente e apenas nos casos mais graves da deficiência. Nesta última medida, são procedimentos úteis para identificar a severidade da desnutrição.

A anamnese alimentar na DPC é instrumento valioso para a determinação da etiologia da desnutrição, sendo sobretudo útil na orientação das ações terapêuticas de recuperação nutricional. Não fornece, entretanto, indicação confiável de diagnóstico. Entre outros motivos, porque a quantificação do consumo alimentar e o estabelecimento das necessidades nutricionais do individuo são procedimentos de grande complexidade e habitualmente sujeitos à excessiva imprecisão.

Em nosso meio - e possivelmente na maioria dos paises não desenvolvidos - a avaliação antropométrica do estado nutricional infantil vem sendo tradicionalmente executada a partir da observação do peso da criança diante de um dado padrão antropométrico de referência. De modo tal que diagnósticos de normalidade são indicados quando o peso observado representa pelo menos $91 \%$ do peso médio esperado para a idade e sexo, e diagnósticos de desnutrição de $1^{\circ}, 2 \%$ e $3^{\circ}$ graus são indicados quando aquele peso representa, respectivamente, 76 a $90 \%, 61$ a $75 \%$ e menos do que $61 \%$ do peso médio esperado. Esta classificação do estado nutricional é internacionalmente conhecida como "Classificação de Gomez", ainda que originalmente Gomez não a tenha proposto exatamente como uma classificação do estado nutricional de indivíduos, mas tão somente como uma forma de classificar crianças desnutridas quanto a gravidade e prognóstico 2 .
Duas ordens básicas de modificaçōes vêm sendo recentemente propostas com relação à avaliação antropométrica do estado nutricional: 1) a adoção de novos padrões antropométricos de reterência em substituição ao tradic.onal padrão de Harvard ${ }^{8}$; 2) a adoção de novos critérios antropométricos em substıtuıção aos "critérios de Gomez".

Tais propostas vêm sendo veiculadas em publıcaçōes internacionais ${ }^{3,9}$ e nacionais ${ }^{4,6}$ de grande alcance, ensejando, nesta medida, um clima propícı à sua efetivação. Como se pretende evidenciar neste artigo, a modıficação de padrão, ainda que pertinente e destinada a ser consensualmente aceita a relativo curto prazo, tem caráter essencialmente académico e reduzidas consequiências práticas. Já a modificação de critérios tem caráter mais complexo implicando importantes conseqüências que requerem cuidadoso juízo antes que se decida pela modificação.

\section{MUDANÇA NO PADRAO ANTROPOMETRICO}

A adequação do padrão antropométrico de rcferência é ponto crucial na avaliação do estado nutricional por meio da observação do crescimento, sendo tanto mais adequado o padrão quanto mais ele se aproximar da condição de padrão de normalidade da população examinada. Esta aproximação, por outro lado, depende basicamente do cumprimento de três condições:

1. o potencial de crescimento dos indivíduos da população de referência deve reproduzir o potencial de crescimento dos indivíduos da população examinada;

2. os indivíduos da população de referência devem viver em ótimas condiçōes ambientais e, portanto, devem desenvolver plenamente seu potencial de crescimento;

3. os valores antropométricos assinalados no padrão devem reproduzir fidedignamiente a distribuição das medidas do 
MONTEIRO, C.A. Recentes mudanças propostas na avaliação antropométrica do estado nutricional infantil: uma avaliaçăo crítica. Rev. Saúde públ., S. Paulo, 18:56-63, 1984.

conjunto dos indivíduos da população de referência.

Em nosso meio ${ }^{4,6}$, dois padrões antropoInètricos vêm sendo sugeridos para suceder o tradicional padrão Harvard, cuja construção remonta as medidas de crianças brancas de Boston náscidas ainda na primeira metade do século. São os padrões Santo Andre Classe IV 5 e NCHS 7, ambos construídos a partir de estudos realizados nas décadas de 60 e 70 , o primeiro em amostras de famílias de alto nível sócio-econômico do município de Santo André, no Estado de São Paulo, e o segundo em amostras de famílias americanas.

Em relação às duas primeiras condições que determinam a adequação do padrão na avaliação nutricional — identidade de potencial de crescimento entre populaçáo de reterência e população examinada e pleno desenvolvimento do potencial de crescimento da população de referência - os novos padroes propostos nada parecem acrescentar a) padrão Harvard, podendo os três serem considerados alternativas igualmente razoáveis em nosso meio. Isto em função do elevado nivel sócio-econômico de suas respectivas populações de referência e das evidências atualmente disponiveis de que grupos populacionais de origens muito diversas - inclusive e particularmente brancos e negros — tendem a apresentar ritmos de crescimento bastante semelhantes quando são adequadas suas condiçōes de vida ${ }^{1}$.

Apenas em relação à terceira condiçāo a capacidade de o padrão traduzir fidedignamente a distribuição das medidas observadas no conjunto dos indivíduos da população de referência - é que se pode esperar um melhor desempenho dos novos padrões. Tal fato decorre de os mesmos terem utilizado procedimentos metodológicos mais apropriados na definição da amostragem, na padronização das medições e no estabelecimento de curvas. São aspectos, portanto, ligados á metodologia de construção - e não à urigem da população - aqueles que recomendam a substituição preconizada.
Ainda assim, em termos práticos, a proximidade existente entre os valores assinalados com que sejam reduzidas as implicações nos novos padrões e no padrão Harvard faz práticas decorrentes da substituição proposta.

$\mathrm{Na}$ medida em que os dois novos padrões propostos - Santo André Classe IV e NCHS - atendem de maneira semelhante as condiçōes centrais de adequação do3 padrōes, a escolha de um deles dependerá exclusivamente da apreciação que se faça, em cada circunstância, de determinadas características marginais dos referidos padröes. Assim em determinados estudos onde a comparabilidade de resultados for essencial, poderá ser mais vantajoso utilizar-se o padrão NCHS que é o padrão internacional recomendado pela Organização Mundial de Saúde 10. Em outras situações, como no controle rotineiro do crescimento feito em unidades sanitárias, a maior familiaridade com o pádrão Santo André Classe IV, por parte daqueles que irão aplicá-lo, poderá ser decisiva para sua adoção.

\section{MUDANCA NO CRITERIO ANTROPOMETRICO}

O diagnóstico presuntivo da desnutrição efetuado a partir do exame antropométrico baseia-se fundamentalmente no encontro de medidas que, sendo suficientemente baixas, sejam de ocorrência improvável em indivíduos bem nutridos. Conforme já mencionado, a operacionalização deste conceito vem se fazendo tradicionalmente pela aplicação da "Classificação de Gomez" que indica como desnutridos as crianças com pesos equivalentes ou inferiores a $90 \%$ do peso médio esperado para idade e sexo. Contra este critério levantam-se três ordens básicas de objeçōes: a) a inconstante especificidade do diagnóstico nas várias idades; b) a especificidade reduzida do diagnóstico; c) a não discriminação entre formas atuais e pregressas de desnutrição. A partir destas objeções, diversas mudanças vêm sendo propostas no critério antropométrico 
MONTEIRO, C.A. Recentes mudanças propostas na avaliação antropométrica do estado nutricional infantil: uma avaliaçăo crítica. Rev. Saúde públ., S. Paulo, 18:56-63, 1984.

indicativo da desnutrição. A seguir, pretende-se analisar a fundamentação das objeções mencionadas e examinar a propriedade das modificações sugeridas.

A primeira objeção é seguramente a menos controvertida. De fato, a especificidade da "Classificação de Gomez", ou seja, sua capacidade em não atribuir diagnóstico de desnutrição a indivíduos normais, não é constante, uma vez que nas diferentes idades são normalmente esperadas diferentes proporções de indivíduos com pesos equivalentes ou inferiores a $90 \%$ da média. Esta condição é facilmente identificada entre indivíduos normais pela simples inspeção dos padrões antropométricos já mencionados. A partir do padrão Santo André Classe IV 5 , por exemplo, verifica-se que até os 4 anos de idade, cerca de $20 \%$ de crianças normais terão pesos equivalentes ou inferiores a $90 \%$ da média. Dos $\mathbf{5}$ aos 10 anos, na mesma situação, são esperadas cerca de $30 \%$ das crianças, e dos 10 aos 12 anos já serão cerca de $40 \%$ as crianças naquela situação. Desta maneira, neste exemplo, a especificidade do diagnóstico teria variado nas três faixas etárias de 80 para 70 para $60 \%$.

A alternativa para que o diagnóstico da anormalidade antropométrica seja efetuado sempre com a mesma especificidade consiste em se definir o nível crítico do indicador antropométrico como um determinado percentil do padrão de referência. No caso específico, o procedimento recomendado consistiria em substituir o critério peso equivalente ou inferior a $90 \%$ da média esperada pelo critério peso equivalente ou inferior a um dado percentil do padrão de referência. Caso se adotasse, por exemplo, o percentil 20 do padrão, ter-se-ia, em todas as idades, o diagnóstico efetuado com $80 \%$ de especificidade, pois que, em todas as idades, apenas $20 \%$ de indivíduos normais teriam pesos equivalentes ou inferiores ao nivel crítico utilizado.

Outra alternativa para garantir especificidade constante no diagnóstico da anorma- lidade antropométrica seria a adoção de niveis críticos baseados em afastamentos da média quantificados em unidades de desvio-padrảo. Esta alternativa apenas é inteiramente pertinente quando é normal a distribuição da medida na população de referência, fato que se observa para a medida da altura, mas que não se observa para a medida do peso.

A segunda objeção levantada contra a "Classificação de Gomez" refere-se à reduzida especificidade da mesma no diagnóstico da desnutrição. De fato, como pôde ser visto nos exemplos, anteriores, a especificidade da Classificação não passaria de $80 \%$ até os 4 anos de idade e seria ainda menor nas idades subseqüentes.

Em relação a este aspecto, a mudança sugerida consiste em se rebaixar o limite ponderal indicativo da desnutrição, pois, como em qualquer outra classificação antropométrica, o diagnóstico da anormalidade ganha maior especificidade à medida que é menor o nível crítico adotado. No caso particular, o que vem se propondo é a adoção de níveis críticos que garantam especificidades próximas a $100 \% 3,6,8,10$. Para tanto, seriam consideradas desnutridas apenas as crianças com pesos inferiores ao percentil 3 do padrão ou ainda com pesos situados mais de dois desvios-padrão abaixo da média esperada para idade e sexo. Em ambos os casos, em termos práticos, os critérios propostos implicariam admifir como normais a grande maioria dos desnutridos de primeiro grau da "Classificação de Gomez". Este exato procedimento, ou seja, considerar $75 \%$ do peso médio esperado como o limite ponderal indicativo de desnutrição, já vem sendo usado na prática em alguns programas de recuperação nutricional.

Antes que se possa apreciar devidamente a propriedade desta segunda objeção à "Classificação de Gomez" - e antes portanto de decidir pela eventual aceitação das modificaçōes que dela decorrem - é necessário considerar que, em qualquer classifica- 
MONTEIRO, C.A. Recentes mudanças propostas na avaliação antropométrica do estado nutricional infantil: uma avaliação crítica. Rev. Saúde públ., S. Paulo, 18:56-63, 1984.

ção antropométrica do estado nutricional, acréscimos em especificidade necessariamente significam decréscimos em sensibilidade. Isto porque a mesma redução do nivel crítico que faz diminuir a proporção de "falsos-positivos", faz crescer a proporção de "falsos-negativos". Tal situação determina que o estabelecimento do nível crítico da classificação antropométrica deve considerar simultaneamente os inconvenientes advindos tanto da baixa especificidade quanto da baixa sensibilidade. Para um programa de recuperação nutricional, por exemplo, "falsos positivos" em excesso implicarão diluir desnecessariamente os recursos disponíveis e subestimar a eficácia do tratamento oferecido. "Falsos-negativos", por sua vez, significarão a exclusão de individuos que, em potencial, poderiam ser recuperados pelo programa.

Outro fator adicional que torna complexa a escolha do nivel crítico da classificação antropométrica é a relação que une frequiência da desnutrição, especificidade da classificação e proporção total de indivíduos erroneamente diagnosticados (seja como normais, seja como desnutridos). Em função desta relação, em locais onde é maior a prevalência da desnutrição, é justo admitir-se uma menor preocupação com a especificidade da classificação, uma vez que a população normal - aquela exposta a diagnósticos errôneos de "falsos-positivos" é relativamente pouco numerosa. Situação inversa ocorre em locais com menor prevalência de desnutridos, como nos países desenvolvidos, onde a especificidade do diagnóstico deve ser necessariamente alta sob pena de os "falsos-positivos" superarem em número os verdadeiros desnutridos.

Desta forma, o reconhecimento da relativa baixa especificidade da "Classificação de Gomez" e os inconvenientes dela decorrentes - particularmente na indicação da recuperação nutricional - não são por si suficientes para recomendar em nosso meio o rebaixamento extremo do limite antropométrico indicativo da desnutrição. Entre nós, onde regra geral é endêmica a presença da desnutrição, a adoção de niveis crítions altamente específicos e pouco sensiveis con'o os propostos -percentil 3, média menos dois desvios, adequação de $75 \%$ à mértia -teriam como principal conseqüência a produção de situações onde freqüentemente os "falsos-negativos" (desnutridos não diagnosticados) superariam em numerc os desnutridos efetivamente identificados.

A terceira objeção levantada contra a "Classificação de Gomez" refere-se à não discriminação entre formas atuais e pregressas de desnutrição. De fato, na medida em que a referida classificação se baseia em um déficit já instalado de crescimento, ela nada pode informar quanto ao período de ocorrência da desnutrição nem quanto à eventual persistência do processo que a gerou. Esta limitação, que pode ser considerada de pequena monta em estudos de prevalência, assume particular importância na indicação terapêutica e no acompanhamento individual de desnutridos, pois a reversão dos prejuízos determinados pela desnutrição depende fortemente da precocidade do tratamento oferecido.

A alternativa mais freqüentemente proposta como meio de possibilitar à classificação antropométrica a discriminação entre formas pregressas e atuais de desnutrição consiste em se substituir a análise do peso do indivíduo pela análise simultânea de sua altura e peso: a altura analisada diante da idade e o peso analisado diante da altura efetivamente observada $\mathbf{3 , 6 , 9}$. Nesta análise, déficits exclusivos de altura caracterizariam a desnutrição pregressa, déficits exclusivos de peso para altura caracterizariam a desnutriçăo atual e déficits mistos caracterizariam a desnutrição crônica.

A substituição de indicadores antropométricos contida na modificação proposta tem contra si dois fatores operacionais bastante importantes: a necessidade em se obter 
MONTEIRO, C.A. Recentes mudanças propostas na avaliação antropométrica do estado nutricional infantil: uma avaliação crítica. Rev. Saúde públ., S. Paulo, 18:56-63, 1984.

adicionalmente a medida da altura - procedimento distante de ser simples nos primeiros anos de vida - e a dificuldade em se dispor de um padrão adequado de peso para altura. Em relação a este último aspecto, não são disponíveis padrões nacionais e a utilização de padrões internacionais é complexa dada a excessiva influência que fatores étnicos e culturais podem exercer sobre a relação peso/altura. Outro aspecto que limita a modificação proposta se refere à subestimação dos quadros atuais de desnutrição principalmente entre crianças pequenas que podem responder à insuficiência alimentar reduzindo uniformemente sua velocidade de crescimento e conservando suas proporções antropométricas. Em relação a crianças pequenas em fase tápida de crescimento, deve-se acrescentar às considerações já efetuadas o fato de que a observação a curtos intervalos do indicador peso/idade pode representar alternativa mais factivel e mais precisa para discriminar quadros atuais e quadros pregressos de desnutrição.

Do exposto evidencia-se ser mais prudente entender os novos indicadores propostos - altura/idade e peso/altura como complementos que, em determinadas situações, poderiam acrescentar informações àquelas fornecidas pela aplicação do tradicional indicador peso/idade.

Revendo em conjunto as objeções mais freqüentemente levantadas contra o método tradicional pelo qual vem se fazendo o diagnóstico antropométrico da desnutrição infantil, bem como as modificações que recentemente têm sido propostas como forma de aperfeiçoar aquele diagnóstico, verifica-se que:

- a adoção de novos padrões antropométricos ("Santo André Classe IV" e "NCHS") está plenamente recomendada, ainda que em termos práticos não se devam esperar resultados muito diferentes daqueles obtidos com 0 padrão Harvard;
- a adoção de valores críticos baseados em percentis do padrão de referência - e não em afastamentos percentuais tixos da média - é um importante aperfeiçoamento para o diagnóstico antropométrico, particularmente quando se examina um amplo espectro de idades e/ou quando se cogita utilizar mais de um indicador;

- a preocupação unilateral com a especificidade do método diagnóstico e a conseqüente proposta de rebaixamento extremo de níveis críticos não fazem sentido em situações como as de nosso meio, onde o caráter endêmico da desnutrição reclama uma preocupação no mínimo simétrica com a sensibilidade;

- a substituição de indicadores - alturai idade e peso/altura em vez de peso/ idade -, proposta como forma de dotar a classificação antropométrica de poder discriminatório quanto à natureza atual ou pregressa da desnutrição, tem contra si importantes fatores operacionais além de, em determinadas situações, implicar em precisão menor do que a que seria obtida acompanhando-se $o$ indicador peso/idade.

Da análise realizada, depreende-se também que a propriedade dos critérios antropométricos utilizados para o diagnóstico da desnutrição não pode ser devidamente apreciada sem que se configure o propósito da avaliação nutricional e sem que se considere o meio específico onde ela será aplicada.

$\mathrm{Na}$ situação concreta de nosso meio, onde a desnutrição está distante de poder ser considerada um evento raro, dois propósitos distintos da avaliação antropométrica devem ser individualizados: a estimativa da prevalência da desnutrição na população e a avaliação nutricional individual.

$\mathrm{Na}$ avaliação feita a nível populacional, não parece haver maiores inconvenientes em 
MONTEIRO, C.A. Recentes mudanças propostas na avaliação antropométrica do estado nutricional infantil: uma avaliação crítica. Rev. Saúde públ., S. Paulo, 18:56-63, 1984.

seguir empregando em nosso meio o indicador peso/idade e os tradicionais critérios da "Classificação de Gomez" que definem a desnutrição de $1^{\circ}, 2^{\circ}$ e $3{ }^{\circ}$ graus. A partir de cinco anos de idade, para obedecer a crescente variabilidade do peso diante da idade, eventualmente poder-se-ia trabalhar com menores adequações percentuais críticas que seriam determinadas de modo a aproximar a especificidade do diagnóstico nas várias idades. Maiores modificações não seriam recomendáveis, pois as vantagens marginais que trariam não seriam suficientes para contrabalançar as desvantagens, particularmente no que se refere a interpretação e comparabilidade de resultados.

$\mathrm{Na}$ avaliação de indivíduos - quando o propósito da avaliação nutricional é o de necessariamente estabelecer prognósticos, indicar terapêuticas e avaliar recuperação
- são evidentemente maiores as deficiências dos critérios antropométricos sustentados pela "Classificação de Gomez", estando, neste caso, plenamente justificada a busca de critérios alternativos que, desde já deverão prever:

- a adoção de percentis para expressar valores antropométricos críticos;

- o estabelecimento de níveis críticos que garantam ao diagnóstico antropométrico uma especificidade mínima definida previamente e de acordo com o objetivo do diagnóstico e a magnitude da desnutrição rio local;

- a observação antropométrica continuada em complemento à observação instantânea.

Em futura publicação, pretendemos voltar ao tema com uma proposta concreta de critérios antropométricos apropriados para diferentes usos em nosso meio.

MONTEIRO, C. A. [Recently proposed changes in anthropometric evaluation of nutritional status of infants: a critical assessment]. Rev. Saúde públ., S. Paulo, 18:56-63, 1984.

ABSTRACT: Some changes in the evaluation of nutritional status through anthropometry have recently been proposed in prestigious national and international publications. These changes consist of the substitution of "Harvard" anthropometric standards and of the weight/age criteria of the "Gomez Classification". The objections to the traditional methodology and the new proposals are critically analysed in the light of the theory which favours the anthropometric evaluation of nutritional status. From the analysis it is evident that: a) the adoption of new anthropometric standards ("Santo André Classe IV" e "NCHS") is fully justified, although little effect is to be expected from it; b) the adoption of standard percentiles to express cut-off points is an important step forward when a wide age-range is under examination or more than one anthropometric indicator is involved; c) the extreme lowering of cut-off points, and the underlying unilateral concern with diagnostic specificity are not justifiable in our context where malnutrition is not a rare event; d) at some ages, the continuous observation of weight/age may result in greater advantages than the introduction of new indicators such as height/age or weight/height. Also it is evident that the appropriateness of anthropometric criteria in the evaluation of nutrional status can not be properly appraised without the explicitation of the purposes of the evaluation and of the endemic level of malnutrition in the population under examination.

UNITERMS: Anthropometry. Children. Nutritional status. Malnutrition. 
MONTEIRo, C.A. Recentes mudanças propostas na avaliação antropométrica do estado nutricional infantil: uma avaliação crítica. Rev. Saúde públ., S. Paulo, 18:56-63, 1984.

\section{REFERENCIAS}

1. EVELETH, P.B. \& TANNER, J.M. Worldwide variation in human growth. London, Cambridge University Press, 1976.

2. GOMEZ, F. et al. Mortality in second and third degree malnutrition. $J$. trop. Pediatr. environ. Child Hith, 2:77-83, 1956.

3. GUERI, M. et al. La clasificación de Gomez: ha llegado el momento de cambiar? Bol. ofic. sanit. pamamer., 91:540-6, 1981.

4. MARCONDES, E. Normas para o diagnóstico e a classificação dos distúrbios do crescimento e da nutrição-última versão. Pediatria, S. Paulo, 4:307-26, 1982.

5. MARQUES, R.M. et al. Crescimento $e$ desenvolvimento pubertário em criancas $e$ adolescentes brasileiros. II - Altura e peso. São Paulo, Editora Brasileira de Ciências, 1982.

6. MINISTERIO DA PREVIDENCIA E ASSISTENCIA SOCIAL. Manual para

\section{EIBLIOGRAFICAS}

avaliação antropométrica do estado nutricional de crianças até 7 anos de idade. Brasilia, 1983.

7. MONTHLY VITAL STATISTICS REPORT. Hyattsville, Md, 25(3 suppl.):1-22, 1976.

8. STUART, H.C. \& STEVENSON, S.S. Grow th and development. In: Nelson, W.E., ed. Textbook of pediatrics. London, Saunders, 1959.

9. WATERLOW, J.C. et al. The presentation and use of height and weight data for comparing the nutritional status of groups of children under the age of 10 year. Bull. Wld Hith Org., 55:489-98, 1977.

10. WORLD HELTH ORGANIZATION. A growth chart for international use in maternal and child health care. Geneva. 1978.

Recebido para publicacão em 23/09/1983 Aprovado para publicasão em 24/10/1983 